- News \& Views •

\title{
FY-3E: The First Operational Meteorological Satellite Mission in an Early Morning Orbit
}

\author{
Peng ZHANG*, Xiuqing HU, Qifeng LU, Aijun ZHU, Manyun LIN, Ling SUN, Lin CHEN, and Na XU \\ Innovation Center for Fengyun Meteorological Satellite, National Satellite Meteorological Center, \\ China Meteorological Administration, Beijing 100081, China
}

(Received 4 August 2021; revised 26 August 2021; accepted 30 August 2021)

\begin{abstract}
Fengyun-3E (FY-3E), the world's first early-morning-orbit meteorological satellite for civil use, was launched successfully at the Jiuquan Satellite Launch Center on 5 July 2021. The FY-3E satellite will fill the vacancy of the global early-morning-orbit satellite observation, working together with the FY-3C and FY-3D satellites to achieve the data coverage of early morning, morning, and afternoon orbits. The combination of these three satellites will provide global data coverage for numerical weather prediction (NWP) at 6-hour intervals, effectively improving the accuracy and time efficiency of global NWP, which is of great significance to perfect the global earth observing system. In this article, the background and meteorological requirements for the early-morning-orbit satellite are reviewed, and the specifications of the FY-3E satellite, as well as the characteristics of the onboard instrumentation for earth observations, are also introduced. In addition, the ground segment and the retrieved geophysical products are also presented. It is believed that the NWP communities will significantly benefit from an optimal temporal distribution of observations provided by the early morning, mid-morning, and afternoon satellite missions. Further benefits are expected in numerous applications such as the monitoring of severe weather/climate events, the development of improved sampling designs of the diurnal cycle for accurate climate data records, more efficient monitoring of air quality by thermal infrared remote sensing, and the quasicontinuous monitoring of the sun for space weather and climate.
\end{abstract}

Key words: FY-3E, operational meteorological satellite, early morning orbit

Citation: Zhang, P., X. Q. Hu, Q. F. Lu, A. J. Zhu, M. Y. Lin, L. Sun, L. Chen, and N. Xu, 2022: FY-3E: The first operational meteorological satellite mission in an early morning orbit. Adv. Atmos. Sci., 39(1), 1-8, https://doi.org/ 10.1007/s00376-021-1304-7.

\section{Introduction}

Fengyun-3E (FY-3E), the world's first early-morning-orbit meteorological satellite for civil use, was launched successfully at the Jiuquan Satellite Launch Center on 5 July 2021. FY-3E is the fifth satellite, among the second generation of the Chinese meteorological satellites, in a near-polar, sun-synchronous orbit (hereafter referred to as the polar; Zhang et al., 2018). Its primary mission is to provide global observations for numerical weather prediction (NWP) (Zhang et al., 2015). Furthermore, it will also benefit the fields of weather, climate, and environmental science due to its multiple instruments, global coverage, and high spatial resolution.

The polar-orbiting satellites synchronized their ascending nodes (the point where the satellite crosses the equatorial plane and goes northward) with the sun so that they can cross the equator at the same local solar time (LST) every day in a north-south-oriented ellipse (Kidder and Vonder Haar, 1995). The polar-orbiting satellites are classified by orbits that pass at different LSTs. Mid-morning satellites (AM) ascend (or descend) at around 10 LST, afternoon satellites (PM) at around 14 LST, and early-morning satellites (EM) at around 6 LST (as shown in Fig. 1). The ground track of EM satellites is near the terminator line, i.e., the dividing line which distinguishes the illuminated face of the sunlit Earth and its night side. Considering that the polar-orbiting meteorological satellites are extremely important to global NWP models, the baseline configuration of the core polar operational constellation has evolved from a two-orbit system (mid-morning and afternoon orbits) to a three-orbit system (early-morning, mid-morning, and afternoon orbits) following the World Meteorological Organization

\footnotetext{
* Corresponding author: Peng ZHANG

Email: zhangp@cma.gov.cn
} 


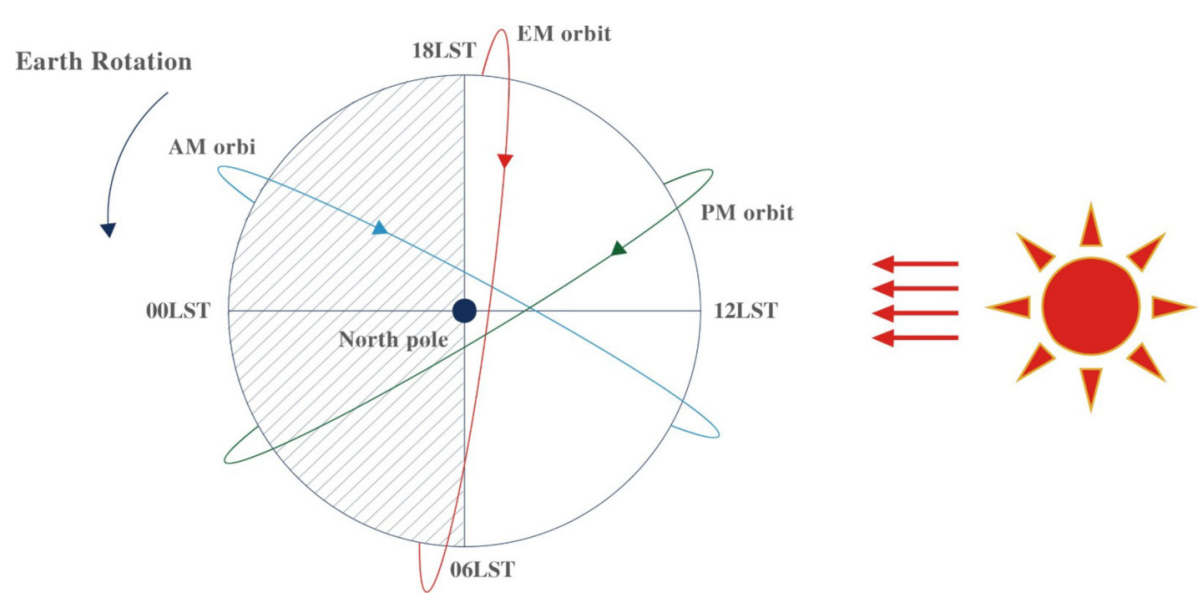

Fig. 1. The schematic diagram for orbital planes of the three polar-orbiting satellites over the Northern Hemisphere. The EM orbit in red is near the limit between the illuminated side and the night side of the Earth.

(WMO) "Vision for global observing systems in 2025" (WMO, 2009).

\section{Meteorological requirements}

Eyre and Lorenc (1989) proposed the direct use of satellite-sounding radiances in the NWP. Four years later, Eyre et al. (1993) successfully assimilated the radiation information provided by the TIROS Operational Vertical Sounder into the NWP system through a one-dimensional variational analysis. Eyre's work has launched a new era of using the polar-orbiting meteorological satellite data into the NWP model. Joo et al. (2013) found that satellite observations account for $64 \%$ of the short-range forecast error reduction, while the remaining $36 \%$ comes from the assimilation of ground-based observations. Moreover, the polar-orbiting satellite data contributes to around $90 \%$ of the satellite observation-involved forecast error reduction. Currently, the European Centre for Medium-Range Weather Forecasts (ECMWF) has already input hundreds of satellite observations into its NWP model (Florence et al., 2018).

The current global NWP model assimilates satellite observations at 6-hour intervals, which requires an initial meteorological field every six hours. If only the AM and PM satellites are deployed in orbits, about $20 \%$ of the global area cannot be covered by satellite observations (Fig. 2). The long-term meteorological satellite observation plans of Europe, the United States of America, and China are expected to provide robust components for the AM and PM orbits, but with an anticipated gap in the EM orbit, according to the WMO (2009) and the Coordination Group for Meteorological Satellites (CGMS, 2011). Table 1 shows the current on-orbit polar operational meteorological satellites, and it is evident that all the satellites are placed in the AM or PM orbit except the latest launch of the FY-3E.

The critical situation above has been highlighted on several occasions by the CGMS and the WMO Commission for Basic Systems. The EM satellites are expected to work synergistically with the AM and PM satellites to provide 100-percent global data coverage for initial meteorological fields in the NWP model every six hours, thereby increasing the forecast accuracy on both hemispheric and regional scales (WMO, 2013).

In 2012, the China Meteorological Administration (CMA) expressed its willingness to investigate the feasibility of conducting a mission with sounding capabilities on the EM orbit to better deploy the atmospheric sounding systems on the planned three orbits (CGMS, 2012). To dedicate particular attention and support to the CMA in the process of assessing such an option, the WMO and the CGMS convened a "Tiger Team" to coordinate the technical evaluation of the global and regional impacts from placing the FY-3 satellite in the EM orbit. A seminar was convened in Beijing on 25 and 26 April 2013 to critically analyze the results from various impact studies conducted by the "Tiger Team". An assessment of the benefits of a satellite mission in an EM orbit has since been drafted and reported (WMO, 2013).

Recognizing the benefits of the EM-orbiting satellites, especially regarding the great significance of obtaining evenly distributed sounding data for global and regional NWP systems, the CMA has drafted the mission requirements of FY-3E in 2014, where the FY-3E is set as one satellite in the FY-3 third phase program. The FY-3 third phase program, consisting of four satellites, was approved and funded by the Chinese government in 2018. As the first satellite in the third phase of the FY-3 series, the FY-3E satellite was originally scheduled to launch in late 2020, before being postponed to 2021 or later. The mission installed 11 onboard instruments to provide global observations for NWP assimilation to improve weather forecast accuracy as well as to monitor other meteorological, oceanographic, and solar-terrestrial physics environments. 


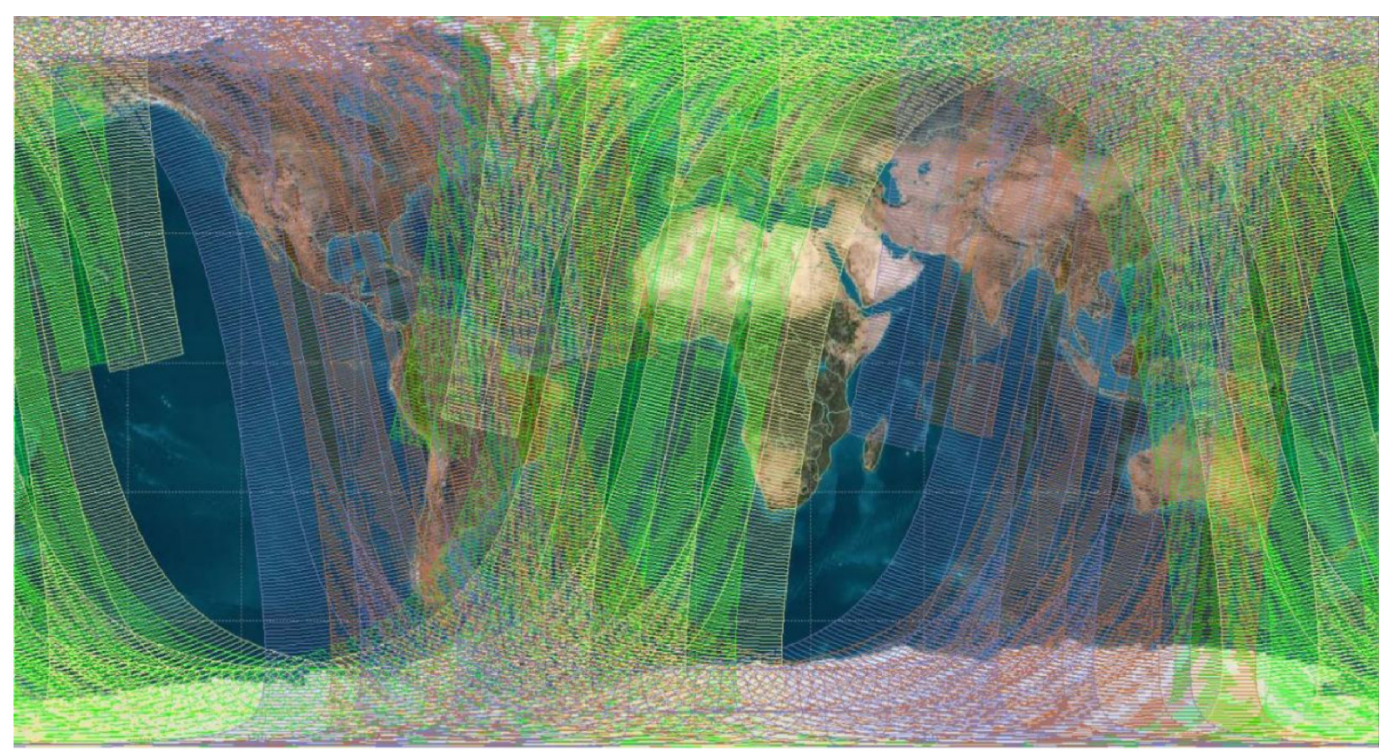

\section{$\begin{array}{llll}\text { FY-3C } & \text { Metop-B/C } & \text { FY-3B/D } & \text { NPP/JPSS-1 }\end{array}$}

Fig. 2. Global coverage per six hours by current on-orbit polar meteorological satellites.

Table 1. Current on-orbit polar meteorological satellites.

\begin{tabular}{cccc}
\hline Number & Satellite name & Orbit attribution & Equator crossing time (ECT) in local standard time \\
\hline 1 & FY-3B & Afternoon & 1445 \\
2 & FY-3C & Mid-morning & 0907 \\
3 & FY-3D & Afternoon & 1329 \\
4 & NPP & Afternoon & 1325 \\
5 & JPSS-1 (NOAA-20) & Afternoon & 1325 \\
6 & METOP-B & Mid-morning & 0930 \\
7 & METOP-C & Mid-morning & 0930 \\
8 & FY-3E & Early-morning & 0530 \\
\hline
\end{tabular}

\section{Satellite platform and instrument configuration}

\subsection{Orbit specifications}

The FY-3E is a sun-synchronous polar-orbiting environmental satellite. It is generally a hexahedron with dimensions of $5.7 \mathrm{~m} \times 3.2 \mathrm{~m} \times 11.6 \mathrm{~m}$ in flight mode, with a total mass of about $2675 \mathrm{~kg}$. One solar panel is fixedly mounted on one side of the satellite's main body. The attitude control of the satellite employs a three-axis stabilization with a measuring precision of $90 \mathrm{~m}$, and an onboard star sensor is employed for attitude sensing. Table 2 illustrates the major orbital parameters of the satellite.

\subsection{Instrument configuration}

There are 11 instruments onboard the FY-3E satellite, as listed in Table 3. Specifically, only one instrument, the Microwave Humidity Sounder-II (MWHS-II), is identical to the one flown on the FY-3D mission. The three brand-new instruments include the dual-frequency wind radar (WindRad), the solar spectral irradiance monitor(SSIM), and the solar X-ray and extreme ultraviolet imager(X-EUVI). Seven improved instruments consist of: the medium resolution spectral imager with low-light capability (MERSI-LL), the microwave temperature sounder (MWTS-III), the hyperspectral infrared atmospheric sounder (HIRAS-II), the global navigation satellite system occultation sounder with a reflectometry technique (GNOS-II), the solar irradiance monitor (SIM-II), the space environment monitor (SEM), and the ionospheric photometer with three view angles (Tri-IPM). Table 3 shows the main characteristics of the onboard instruments. Figure 3 presents the satellite spacecraft model and associated payload configuration. 
Table 2. Orbit specifications of the FY-3E.

\begin{tabular}{cc}
\hline Orbit & Sun-synchronous \\
\hline Orbit Height & $836 \mathrm{~km}$ \\
Inclination $\left(^{\circ}\right)$ & $98.75^{\circ}$ \\
Local time at descending node & $0530-0550 \mathrm{UTC}$ \\
Quasi-repeat time & $5.5 \mathrm{~d}($ design $4 \mathrm{~d}-10 \mathrm{~d})$ \\
Eccentricity & $\leqslant 0.0025$ \\
Orbital maintenances & 20 min $(8 \mathrm{yr})^{-1}$ \\
Satellite life & Design life: $8 \mathrm{yr}$ \\
\hline
\end{tabular}

Table 3. Instruments onboard the FY-3E and their primary characteristics.

\begin{tabular}{|c|c|c|c|}
\hline Instruments & Spectral range & Spatial Resolution & Primary Purpose \\
\hline MERSI-LL & $0.5-12.5 \mu \mathrm{m}$ & $0.25-1 \mathrm{~km}$ & $\begin{array}{l}\text { Large dynamic range imaging, land surface temperature/sea surface } \\
\text { temperature, atmospheric precipitable water }\end{array}$ \\
\hline HIRAS-II & $650-2550 \mathrm{~cm}^{-1}$ & $14 \mathrm{~km}$ & $\begin{array}{l}\text { Numerical weather forecast, atmospheric temperature and humidity } \\
\text { profiles, surface temperature, atmospheric composition, green- } \\
\text { house gases, dust, cloud cover, etc. }\end{array}$ \\
\hline MWHS-II & 89-190 GHz & $15-30 \mathrm{~km}$ & $\begin{array}{l}\text { Atmospheric temperature and humidity profiles, assimilation in } \\
\text { NWP and reanalysis, precipitation detection }\end{array}$ \\
\hline MWTS-III & $23.8-57 \mathrm{GHz}$ & $33 \mathrm{~km}$ & Atmospheric temperature profile \\
\hline GNOS-II & $\begin{array}{l}\text { 1575.42, } 1227.6 \\
1561.098,1268.52 \mathrm{MHz}\end{array}$ & $\begin{array}{l}25 \mathrm{~km}(\mathrm{GNSS}-\mathrm{R}) \\
1-3 \mathrm{~km} \text { (ion-RO), } \\
150-300 \mathrm{~m}(\mathrm{~atm}-\mathrm{RO})\end{array}$ & $\begin{array}{l}\text { Atmospheric profile, wet atmospheric profile, electron density pro- } \\
\text { file, sea surface wind speed }\end{array}$ \\
\hline WindRAD & $5.4,13.256 \mathrm{GHz}$ & $10 \mathrm{~km}, 20 \mathrm{~km}$ & $\begin{array}{l}\text { Sea surface wind vector including wind speed and wind direction, } \\
\text { sea ice }\end{array}$ \\
\hline SIM-II & $0.2-20 \mu \mathrm{m}$ & - & Total solar irradiance \\
\hline SSIM & $165-1650 \mathrm{~nm}$ & - & Solar spectra irradiance \\
\hline X-EUVI & $0.6-8.0 \mathrm{~nm} ; 19.5 \mathrm{~nm}$ & - & $\begin{array}{l}\text { Full disk solar X-ray and ultraviolet images, to enhance the fore- } \\
\text { casts of space weather }\end{array}$ \\
\hline Tri-IPM & $135.6 \mathrm{~nm} ; 150-160 \mathrm{~nm}$ & $32 \mathrm{~km}$ & OI and $\mathrm{N}_{2} \mathrm{LBH}$ airglow radiation intensity \\
\hline SEM-II & $30 \mathrm{ev}-300 \mathrm{MeV}$ & - & $\begin{array}{l}\text { Satellite security designs, scientific studies, development of radi- } \\
\text { ation belt models, space weather monitoring, and disaster warning }\end{array}$ \\
\hline
\end{tabular}

\section{Ground segment for data processing and product generation}

\subsection{Ground segment}

The spacecraft communication links use S-band and X-band microwaves. Commands are given via the S-band only. Commands and telemetry links are active simultaneously. The S-band section of the communication subsystem provides primary telemetry and command service to and from ground stations of the FY-3E, while the X-band section of the communication subsystem provides the science and engineering data downlink for common spacecraft of the FY-3E.

The FY-3E ground segment for data receiving and processing inherits the framework of previous satellites, especially the FY-3D, with enhanced information technology resources and cloud service capabilities.

When the FY-3E is in orbit, it broadcasts the real-time, medium-resolution spectral picture transmission (MPT) data corresponding to all 11 payloads in the X-band to the world. The direct readout service of the FY-3E enables users equipped with proper data acquisition devices to receive data directly from satellite broadcasts. The international pre-processing software package will be provided to support direct broadcasting. The global delayed picture transmission (DPT) data of the 11 payloads in the X-band are transmitted to the ground stations whenever the FY-3E is passing over the acquisition range within the ground station network.

The FY-3E global data acquisition network consists of seven ground stations, including five domestic ground stations (Beijing station, Guangzhou station, Urumqi station, Jiamusi station, and Kashi station, as shown in Fig. 4a) and two high-latitude ground stations (Kiruna station and Troll station, as shown in Fig. 4b). Under the unified scheduling of the integrated operation and control system, the FY-3E data are received and transmitted to the Data Processing Center in Beijing. Global data acquisition latency would be expected with three hours. After a six-month, on-orbit commission test, the FY-3E data will be made available on the Fengyun satellite data service website (http://data.nsmc.org.cn). 


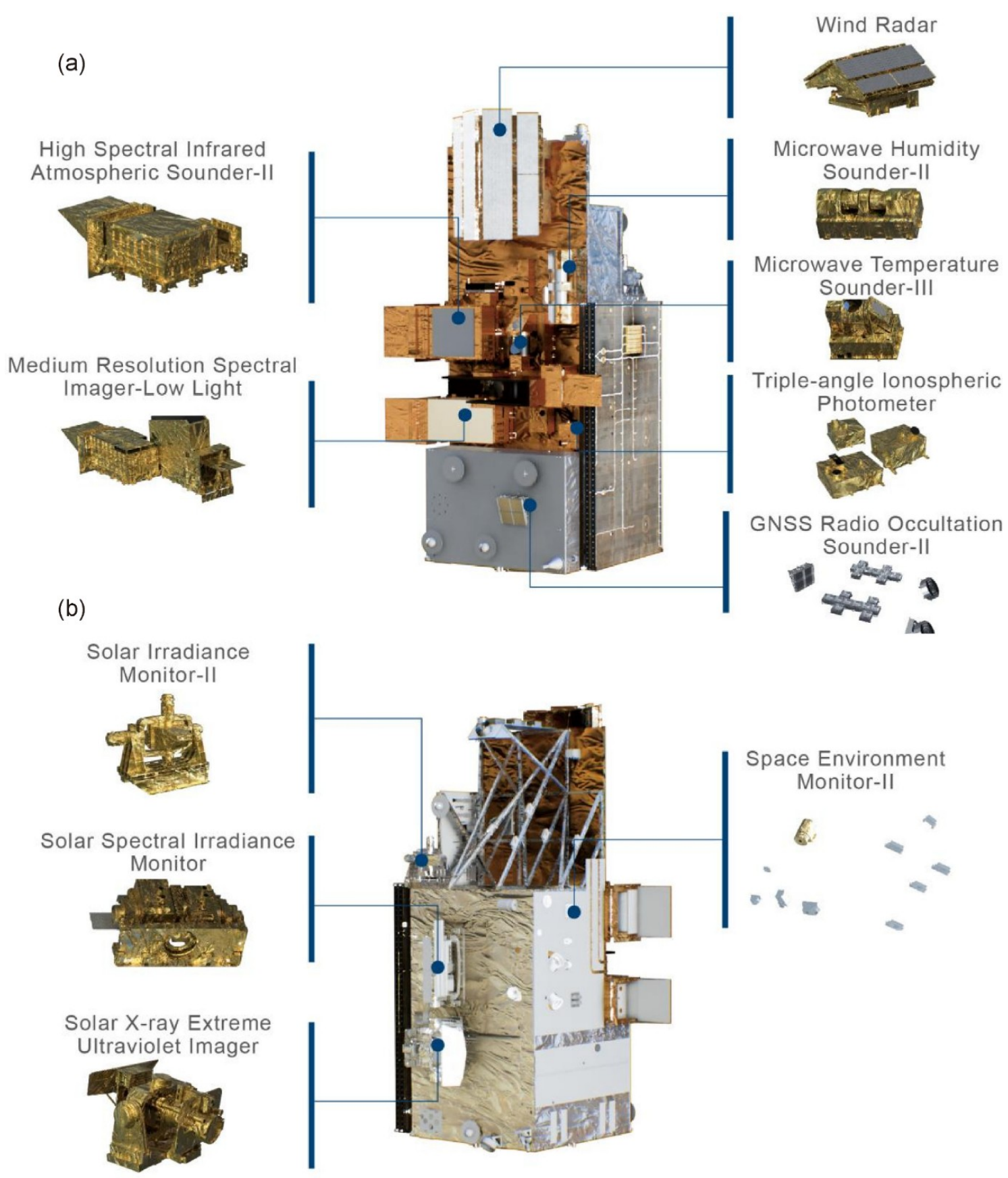

Fig. 3. The satellite spacecraft model and associated payload configuration.

\subsection{Geophysical products}

With multiple sensors onboard the FY-3E, a complete remote sensing product system has been generated, producing more than 40 kinds of products in six categories, including images, clouds and radiation, sea and land surface, atmosphere, atmospheric compositions, and space weather, which cover the main requirements of the WMO Integrated Global Observing System (Table 4). Based on the observation capability of the new early-morning orbit, new remote sensing products, such as the nighttime light imageries with near-constant contrast in low-light conditions, the sea-surface wind field, the sea-ice edge and type, the vertical ozone profile, have been developed.

FY-3E offers certain advantages for monitoring trace gases such as carbon monoxide and ozone in the infrared due to the contrasting temperature between the earth's surface layer and the atmospheric boundary layer on this orbit. The generally smaller cloud amount and lower absolute humidity in the early morning can also provide an advantage for monitoring the air quality.

The early-morning-orbit satellite also offers the potential to observe the sun in an almost continuous manner, provid- 

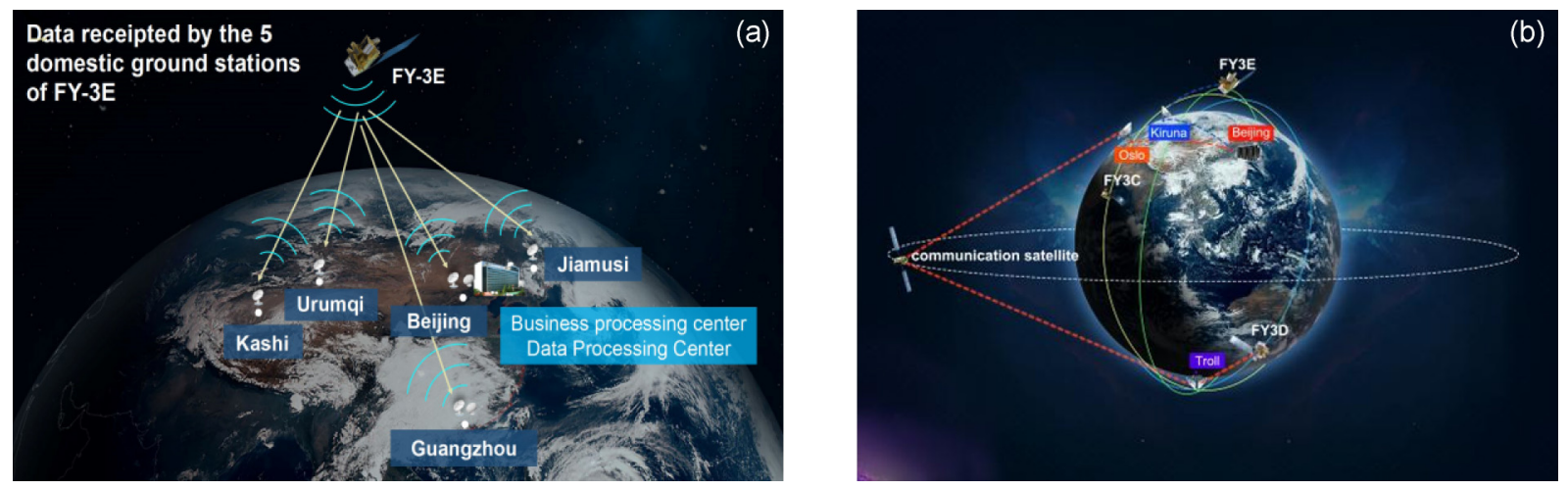

Fig. 4. (a) Five domestic ground stations and (b) two high-latitude ground stations for receiving the FY-3E data.

Table 4. FY-3E product list.

\begin{tabular}{|c|c|}
\hline Instrument & Product \\
\hline \multirow[t]{15}{*}{ Imager/MERSI LL } & Near constant contrast \\
\hline & Nighttime light \\
\hline & Cloud Mask \\
\hline & Cloud amount \\
\hline & Cloud type \\
\hline & Cloud phase \\
\hline & Cloud top temperature \\
\hline & Cloud top height \\
\hline & Cloud top pressure \\
\hline & Total Precipitable Water \\
\hline & Outgoing long-wave radiation \\
\hline & polar Atmosphere Motion Vector \\
\hline & Land surface temperature \\
\hline & Sea Surface Temperature \\
\hline & Snow Cover \\
\hline \multirow[t]{3}{*}{ IR Sounder/ HIRAS } & Cloud-Cleared Radiance \\
\hline & Atmospheric temperature and humidity profiles \\
\hline & Ozone profile \\
\hline \multirow{5}{*}{$\begin{array}{l}\text { MW Sounder / MWHS } \\
\text { GNOS-II }\end{array}$} & Rain Detection \\
\hline & Dry Atmospheric Profile \\
\hline & Wet Atmospheric Profile \\
\hline & Electron Density Profile \\
\hline & Sea surface Wind Speed \\
\hline \multirow[t]{2}{*}{ Scaterometer/ WindRAD } & Ocean Vector Wind (Wind Speed/Wind direction ) \\
\hline & Sea Ice Edge\&Type \\
\hline \multirow[t]{4}{*}{ Solar Irradiance Monitor/SSIM, SIM-II } & Total Solar Irradiance \\
\hline & Spectral Solar Irradiance \\
\hline & TOA incident solar radiation flux \\
\hline & TOA Downwelling Radiation flux \\
\hline \multirow[t]{8}{*}{ Space weather Monitor/SEM } & High Energy Particles \\
\hline & Medium Energy Proton \\
\hline & Medium Energy Electron \\
\hline & Relative Surface Potential \\
\hline & Absolute Surface Potential \\
\hline & Low Frequency Magnetic Field \\
\hline & High Frequency Magnetic Field \\
\hline & Radiation Dose Product \\
\hline \multirow{3}{*}{$\begin{array}{l}\text { Space weather Monitor/Tri-IPM } \\
\text { Space weather Monitor/X-EUVI }\end{array}$} & $\mathrm{O}_{2}$ and $\mathrm{N}_{2}$ Ratio \\
\hline & Solar X-ray Image \\
\hline & Solar Extreme Ultra-violet Image \\
\hline
\end{tabular}


ing significant advantages for monitoring the climate, solar activity, and active regions for space, generating products such as the solar constant, solar spectrum measurements, solar X-ray images, and solar extreme ultraviolet images. The algorithms of the inherited products can been found in Zhang el al.(2009, 2019), Yang et al. (2011, 2012) and Xian et al. $(2021)$

\section{Conclusions}

After about six months of conducting an on-orbit commission test, the FY-3E will transition to operational mode. It will work together with the FY-3C in AM orbit and the FY-3D in PM orbit to constitute the constellation of polar-orbiting satellites in three orbital planes that are distributed roughly 60 degrees apart. The justification for having at least three operational polar-orbiting satellites, rather than two, has been supported by many NWP impact studies over the last decade (Eyre and English, 2008; Bormann and Bauer, 2010; Bormann et al., 2010). The FY-3E, in concert with the FY-3C and the FY$3 \mathrm{D}$, will provide full global coverage in every six-hour data assimilation window. It is expected that the number of onboard FY instruments, which provide the data assimilated into the CMA NWP model GRAPES, will be increased from the current 11 to 18. The amount of the assimilated FY data will increase by 1.5 times compared with the current status. Furthermore, a more uniform temporal spacing of the observations has a particularly significant impact on those cases with rapidly increasing forecast errors, such as rapidly evolving weather systems. Therefore, the FY-3E satellite observations with these practical advantages are extremely important for severe weather situations.

FY-3E increased the local visiting frequency. The EM-AM-PM polar-orbiting constellation can provide up to six measurements per day, approximately every four hours at middle and low latitudes. At high latitudes, the measurements will be more frequent because successive orbits offer overlapping coverage. This polar-orbiting constellation will support a denser sampling of the diurnal cycle, which is critical for monitoring climate factors such as surface temperature, atmospheric temperature, atmospheric humidity, and precipitation.

It is of particular significance at the international level to deploy the FY-3E in the EM orbit. In becoming the primary satellite provider for early morning orbits, China will share a global responsibility with Europe in the mid-morning-orbit observation and with the United States of America in the afternoon-orbit observation. In the follow-up program of the Fengyun 3, there will be another EM orbit satellite that will serve as the successor of FY-3E. According to the roadmap of Fengyun 5 program, an EM orbit satellite also has been planned to keep the continuity of operational measurements. The successful launch of the FY-3E enables the community to meet the baseline configuration agreed upon by the CGMS members in support of the WMO "Vision for global observing systems in 2025".

Acknowledgements. This work was funded by the FY3-03 project and the National Key Technology Research and Development Program of China (Grant Nos. 2018YFB0504900 and 2018YFB0504905).

\section{REFERENCES}

Bormann, N., and P. Bauer, 2010: Estimates of spatial and interchannel observation-error characteristics for current sounder radiances for numerical weather prediction. I: Methods and application to ATOVS data. Quart. J. Roy. Meteor. Soc., 136, 1036-1050, https://doi.org/10.1002/qj.616.

Bormann, N., A. Collard, and P. Bauer, 2010: Estimates of spatial and interchannel observation-error characteristics for current sounder radiances for numerical weather prediction. II: Application to AIRS and IASI data. Quart. J. Roy. Meteor. Soc., 136, 1051-1063, https://doi.org/10.1002/qj.615.

Coordinator Group for Meteorological Satellites (CGMS), 2011: Report of the 39th Meeting of the Coordination Group for Meteorological Satellites. CGMS. [Available from https://www.cgms-info.org/documents/cgms-39-report.pdf.]

Coordinator Group for Meteorological Satellites (CGMS), 2012: Report of the 40th Meeting of the Coordination Group for Meteorological Satellites. CGMS. [Available from https://www.cgms-info.org/documents/cgms-40-report.pdf.]

Eyre, J. R., and A. Lorenc, 1989: Direct use of satellite sounding radiances in numerical weather prediction. Meteor. Mag., 118, $13-16$.

Eyre, J. R, and S. J. English, 2008: Impace studies with satellite data at the Met Office. Proceedings of 4th WMO Workshop on "The Impact of Various Observing Systems on NWP, Geneva, 19-21.

Eyre, J. R., G. A. Kelly, A. P. McNally, E. Andersson, and A. Persson, 1993: Assimilation of TOVS radiance information through onedimensional variational analysis. Quart. J. Roy. Meteor. Soc., 119, 1427-1463, https://doi.org/10.1002/qj.49711951411.

Florence, R., S. J. English, and R. Engelen, 2018: Satellite data assimilation at ECMWF. Proc. 98th American Meteorological Society Annual Meeting, Austin, AMS.

Joo, S., J. Eyre, and R. Marriott, 2013: The impact of Metop and other satellite data within the Met Office global NWP system using an adjoint-based sensitivity method. Mon. Wea. Rev., 141(10), 3331-3342, https://doi.org/10.1175/MWR-D-12-00232.1.

Kidder, S. Q., and T. H. Vonder Haar, 1995: Satellite Meteorology: An Introduction. Academic Press, 466 pp.

World Meteorological Organization (WMO), 2009: The Vision for the Global Observating System in 2025. WMO.

World Meteorological Organization (WMO), 2013: Assessment of the benefits of a satellite mission in an early morning orbit: Report from the WMO-CGMS Tiger Team. WMO. [Available from https://library.wmo.int/index.php?lvl=notice_display\&id14774\#.YS3za 
PkyY18.]

Xian, D., P. Zhang, L. Gao, R. J. Sun, H. Z. Zhang, and X. Jia, 2021: Fengyun meteorological satellite products for earth system science applications. Adv. Atmos. Sci., 38(8), 1267-1284, https://doi.org/10.1007/s00376-021-0425-3.

Yang, J., and C. H. Dong, 2011: New Generation Fengyun Meteorology Satellite Operational Products and Applications. Science Press, 368 pp. (in Chinese)

Yang, J., P. Zhang, N. M. Lu, Z. D. Yang, J. M. Shi, and C. H. Dong, 2012: Improvements on global meteorological observations from the current Fengyun 3 satellites and beyond. International Journal of Digital Earth, 5(3), 251-265, https://doi.org/10.1080/ 17538947.2012.658666.

Zhang, P., J. Yang, C. H. Dong, N. M. Lu, Z. D. Yang, and J. M. Shi, 2009: General introduction on payloads, ground segment and data application of Fengyun 3A. Frontiers of Earth Science in China, 3(3), 367-373, https://doi.org/10.1007/s11707-009-0036-2.

Zhang, P., L. Yang, S. Y. Gu, X. Q. Hu, X. J. Wu, R. H. Wu, Y. M. Bi, and C. Liu, 2015: Meteorological requirements for the early-morning-orbit satellite. Advances in Meteorological Science and Technology, 5(2), 6-12, https://doi.org/10.3969/j.issn.20951973.2015.02.001. (in Chinese with English abstract)

Zhang, P., L. Chen, D. Xian, and Z. Xu, 2018: Recent progress of Fengyun meteorology satellites. Chinese Journal of Space Science, 38(5), 788-796, https://doi.org/10.11728/cjss2018.05.788.

Zhang, P., and Coauthors, 2019: Latest progress of the Chinese meteorological satellite program and core data processing technologies. Adv. Atmos. Sci., 36(9), 1027-1045, https://doi.org/10.1007/s00376-019-8215-x. 